\title{
A comparison between the performance of DWT and LWT in image watermarking
}

\author{
Dujan B. Taha ${ }^{1}$, Taha Basheer Taha², Najla Badie Al Dabagh ${ }^{3}$ \\ ${ }^{1}$ Software Engineering Department, College of Computer Science and Mathematics, Mosul University, Iraq \\ ${ }^{2}$ IT Department, Faculty of Sciences, Tishk International University, Erbil, Iraq \\ ${ }^{3}$ Computer Science Department, College of Computer Science and Mathematics, Mosul University, Iraq
}

\begin{tabular}{l} 
Article Info \\
\hline Article history: \\
Received Aug 25, 2019 \\
Revised Dec 26, 2019 \\
Accepted Feb 3, 2020 \\
\hline
\end{tabular}

\section{Keywords:}

Copyrights protection Discrete wavelet transform Image processing Lifting wavelet transform Transform domain watermark

\begin{abstract}
Image watermarking algorithms can be implemented using time domain or frequency domain-based algorithms. Frequency domain watermarking produces watermarks with higher robustness; hence, many attempts have been proposed in literature using different transformations as DCT, SVD, and DWT. DWT was widely used for many reasons as its spatio-temporal feature, in which the alteration of certain portion will affect only the affected portion. In this paper an experimental comparison between the traditional DWT and the second generation of wavelet which is LWT is initiated. The experimental tests evaluated the performance of both transforms in terms of image quality and watermark robustness.
\end{abstract}

This is an open access article under the CC BY-SA license.

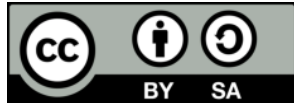

\section{Corresponding Author:}

Taha Basheer Taha,

IT Department, Faculty of Sciences,

Tishk International University, Erbil, Iraq

Email: Taha.Basheer@ishik.edu.iq

\section{INTRODUCTION}

Digital image watermarking is the process of inserting some data as ownership information into images for different purposes as copyright protection, prevent unauthorized duplication and authentication [1]. Insertion of watermark should be done in such a way that the watermark can be completely recovered from the watermarked image. The extraction process becomes more difficult when images are transmitted over a network. Many intentional and unintentional alterations can be applied on it. In such cases, survival of watermark image is desirable [2]. A watermark that survive different attacks is called a robust watermark. Another requirement of the watermark is the invisibility, which is keeping the perceptual quality of the original images high. The watermark is preferred to be totally invisible. Robustness and invisibility are contrary, i.e. increasing the robustness means embedding the watermark with more intensity which decrease the quality of the watermarked image. On the other hand, using low embedding intensity will produce higher quality watermarked images but less robustness. Choosing a suitable threshold of intensity while watermark embedding give a significant effect to the balance between imperceptibility and robustness of the watermarking scheme [3-4]. Watermarking can be implemented as time domain or transform domain processes. Time domain attempts are characterized by simplicity and low computation overhead, but the lack of robustness. Although transform domain watermarking has more complex calculations, it is more robust against attacks that aims to remove or distort the watermark. Frequency domain watermarking is achieved after applying one of time to frequency domain transformations, as singular value decomposition SVD, discrete wavelet transform DWT, and lifting wavelet transform LWT. Discrete wavelet 
transform has some features that make it a good choice for watermarking algorithms, as the feature of spatio-frequency localization in which the particular frequency coefficient of tampering area is only affected when a change is applied on images [5]. Discrete wavelet transform has been used in many attempts in literature. Sweldens [6], proposed a new form of DWT, called Lifting scheme or lifting wavelet transform (LWT), which is called the second generation of wavelet.

Both DWT and LWT were used in image watermarking. DWT was used in many watermarking algorithms as [7] in which the watermark is embedded using DWT with discrete-time chaos system. Another study was performed in [8], where the DWT is used combined with DCT for RGB images. The combination of DWT with DCT is used also in [9] for getting a robust watermarking algorithm. In addition to many other studies where DWT was used and utilized for watermarking [10-14]. LWT on other hand, had been used in recent watermarking attempts. It is combined with SVD in [14, 15], and it is used with support vector machine (SVM) in [16]. Both transforms are commonly used in literature, and both have the same objective which is transform the image into frequency domain coefficients but with different methods and calculations. LWT was also used in [17-21] for watermarking purpose.

In this paper, a comprehensive experiments and evaluation had been initiated to compare the performance for both transforms by embedding the same watermark with the same intensity into similar bands and coefficients in DWT and LWT. And test the quality of the watermarked images and the robustness of the watermarking algorithm on different standard images. The paper is organized as follows; in the next section a theoretical background about both transforms is presented, while in section three the method of watermark embedding is explained, followed by the results of the comparison in section four. The paper is concluded in section five.

\section{THEORITICAL BACKGROUND}

The discrete wavelet transformation can be achieved using filter banks or LWT [22]. A filter bank method, consists of two filters, the low pass filter and the high pass filter followed by down-sampling. An original signal is fed to these filters. The output of filters is the approximation band which contains the important information about the signal, and the details band which consist of high frequency data (Details) in a half size. The inverse process is achieved in reversing the order and restoring the original signal.

For digital images, the filters are applied on rows and columns of each image, accordingly, four bands are created in each DWT decomposition, after applying the low pass filter (L), and high pass filter (H), hence the bands are denoted to as LL, LH, HL, and HH. Figure 1 shows the filter bank method of DWT.

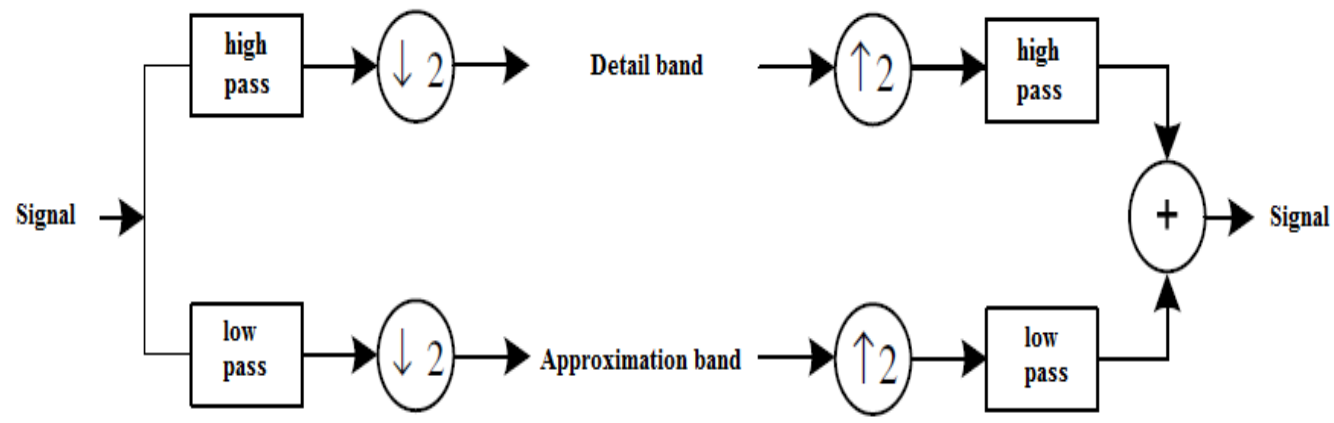

Figure 1. Filter banks

Lifting scheme or lifting wavelet transform LWT was introduced by Swedens [6] provides lower computational process in compare with filter banks used in regular DWT. Furthermore, the Lifting scheme has integer to integer computation where no floating point calculations are required. To produce the approximation and detail bands in LWT, three steps are required: Split: in which the signal is separated into two parts, usually it is split according to the position of the element in the original signal, odd or even. Predict: in this step the details band is created by finding the difference between the original odd sample and the predicted odd sample which is calculated as the average of the two even samples that are adjacent to the odd one. Update: produces the approximation band by updating the values of the average by the difference that is calculated in predict step. Figure 2 shows the LWT process. Inverse of LWT is done in the same way but in reverse order, and by replacing the signs of predict and update steps [22, 23]. 


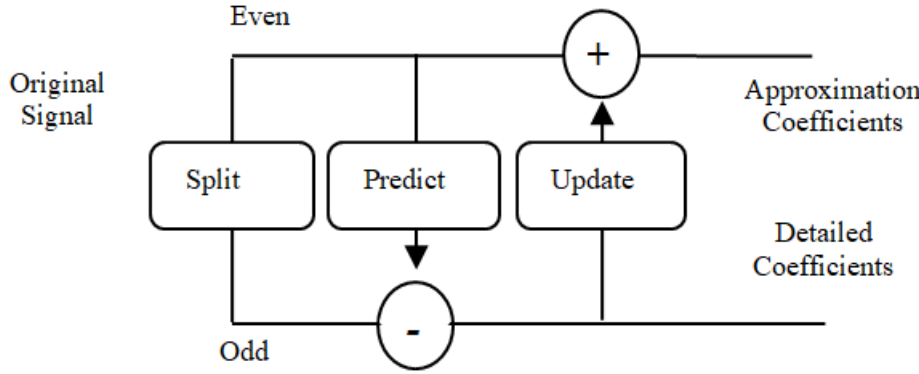

Figure 2. LWT decomposition

Although both transforms divide the signals into details and approximation bands, coefficients within each one are different. Hence, the results of embedding a watermark and recover it may vary, furthermore LWT is reversible while DWT is not. Hence comparisons of both attempts are initiated to evaluate the performance of both transforms using the same embedding algorithm that is explained in the next section.

\section{WATERMARKING PROCESS}

A similar watermarking process is used for both transforms. Below, the embedding and extracting algorithms are explained.

\subsection{Watermark embedding}

DWT and LWT application decomposes the image into four frequency bands; approximation, details, and two middle frequency bands, horizantal and vertical. For both transforms embedding processes, a $512 \times 512$ image was decomposed into four bands each of $256 \times 256$. A wateramrk as shown in Figure 3 of size $64 \times 64$ was embedded after repeating each bit four times to fill one of the decomposed bands and to achieve more robustness. The horizantal middle frequency band was chosen as the middle freuency band has the trade-off between robustness and invisibility when watermarking algorithms are applied. Figure 4 shows the embedding process.

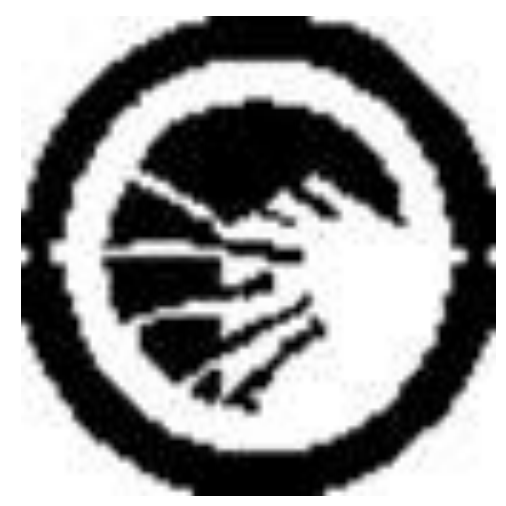

Figure 3. Binary watermark

\subsection{Watermark extraction}

The extracting algorithm is based on the comparison between the original and watermarked bands. After applying the transform (DWT/LWT) on both of the original and watermarked images, each coefficient of the watermarked middle frequency band is compared with its alternative in the original image band. The difference decide the watermark bit. Figure 5 shows the extraction process. It should be noticed that the purpose of the used watermaring algorithms is the comparison. Hence, a simple embedding is used for this purpose. For both transforms, Daubachies mother wavelet was used. 


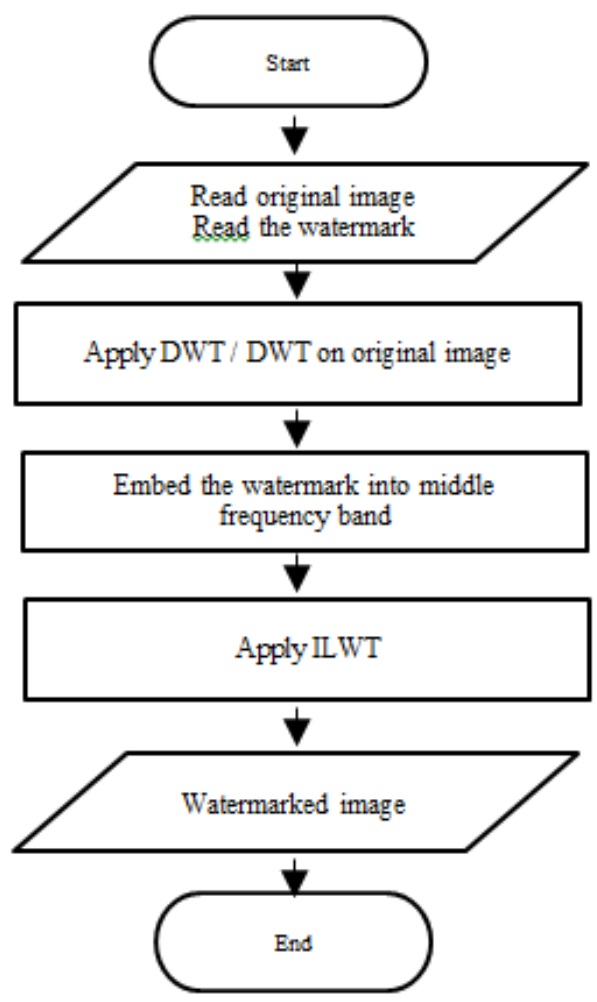

Figure 4. Watermark embedding process

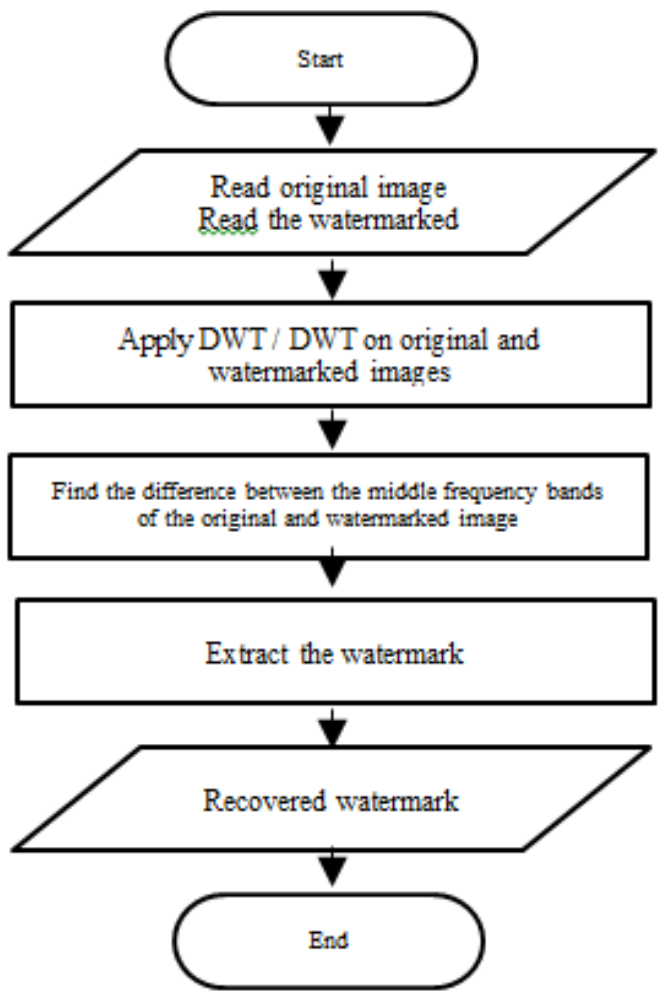

Figure 5. Watermark extraction process

\section{RESULTS}

In this section, the performance evaluation for DWT and LWT in watermarking are tested and compared. The first subsection calculates the peak signal to noise ratio (PSNR) for both transforms and compare them. The next section tests and compares the robustness for the watermark after embedding in both transforms.

\subsection{Perceptual quality evaluation}

The quality of the watermarked images is evaluated for DWT embedding for ten tested images. PSNR results are shown in Table 1. For SSIM [24], which consider the human vison system sensitivity and has better estimation than PSNR [25, 26], the results was equal to 1 for all images except I10 in DWT it was 0.99 , due to the embedding in middle frequency area with low intensity.

For the same tested images, PSNR is calculated for watermarked images using LWT. Table 2 shows the results. Different original and watermarked images according to DWT and LWT transforms are shown in Figure 6. Figure 7 shows the comparison between the two methods for the tested images. It is noticed that the PSNR for images that were watermarked using LWT is higher than its value for DWT based watermarked algorithms.

Table 1. PSNR for DWT-based watermarked images

\begin{tabular}{cc}
\hline Image & PSNR \\
\hline I1 & 48.1326 \\
I2 & 48.1314 \\
I3 & 48.1345 \\
I4 & 48.1308 \\
I5 & 48.1308 \\
I6 & 48.1308 \\
I7 & 48.1308 \\
I8 & 48.1466 \\
I9 & 48.1345 \\
I10 & 48.1708 \\
\hline
\end{tabular}

Table 2. PSNR for LWT-based watermarked images

\begin{tabular}{cc}
\hline Image & PSNR \\
\hline I1 & 49.3794 \\
I2 & 49.3746 \\
I3 & 49.3835 \\
I4 & 49.3751 \\
I5 & 49.3760 \\
I6 & 49.3764 \\
I7 & 49.3745 \\
I8 & 49.3898 \\
I9 & 49.3799 \\
I10 & 49.4162 \\
\hline
\end{tabular}



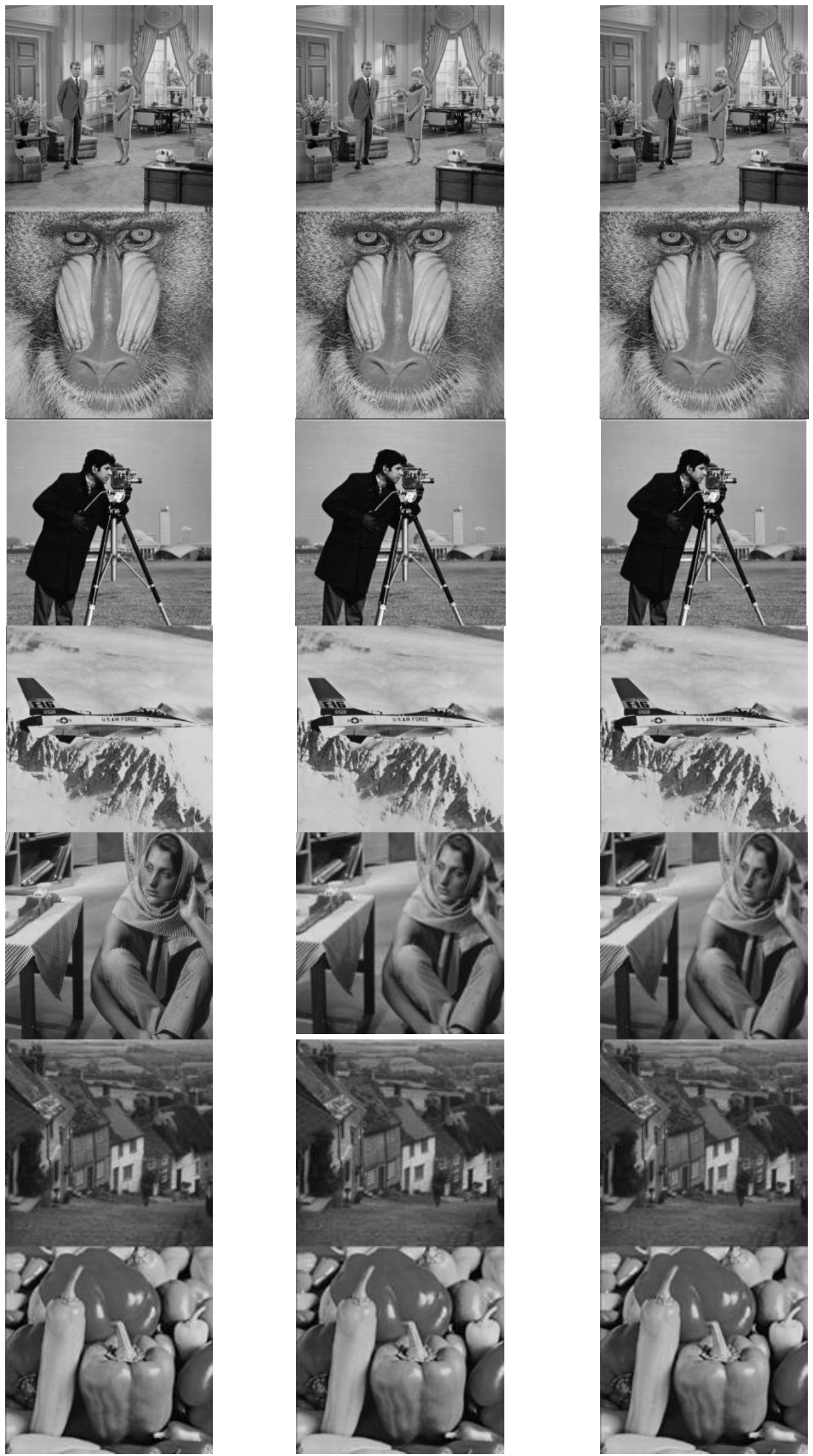

Figure 6. Original images (left), DWT-watermarked (center), LWT-watermarked (right) 

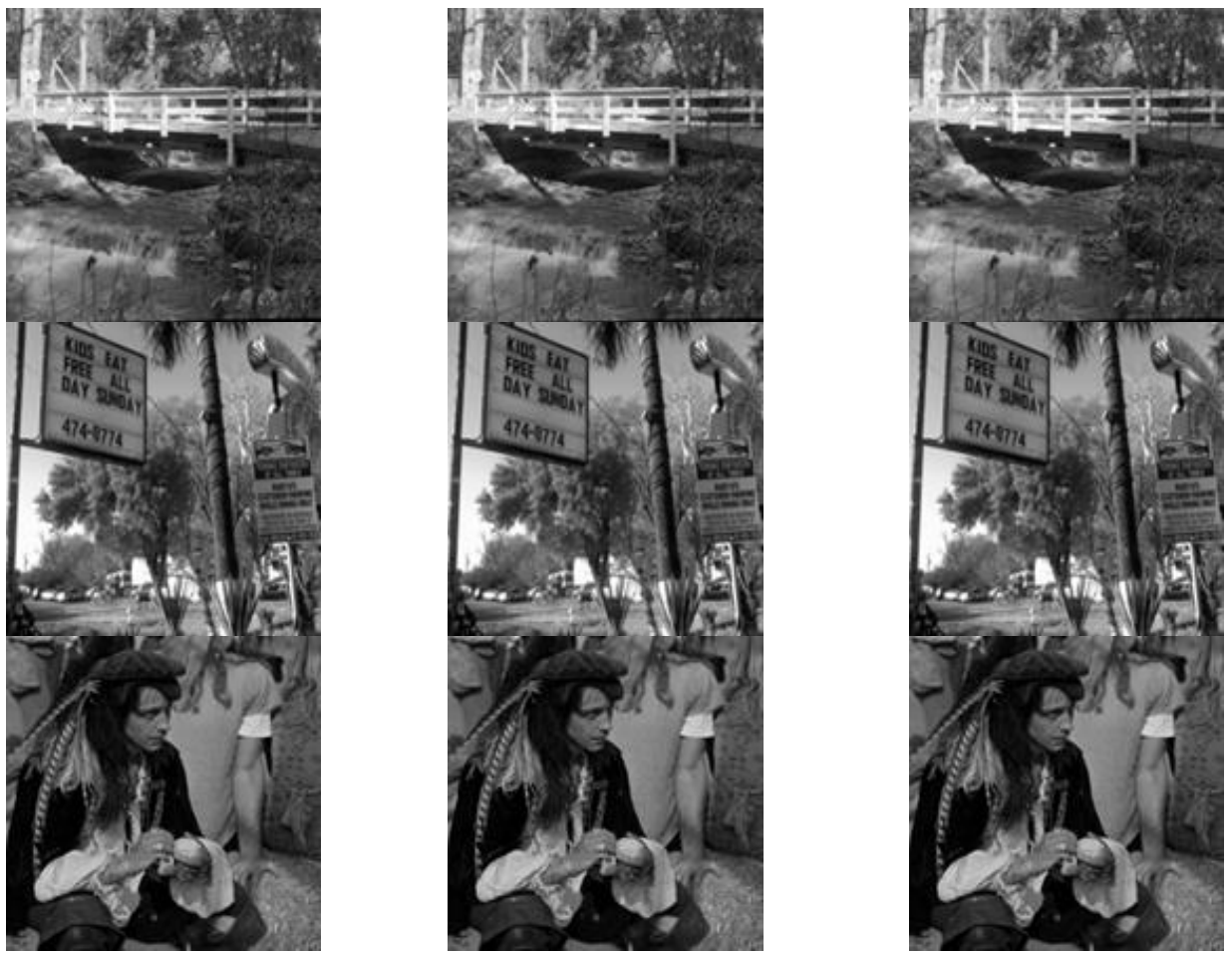

Figure 6. Original images (left), DWT-watermarked (center), LWT- watermarked (right) (continue)

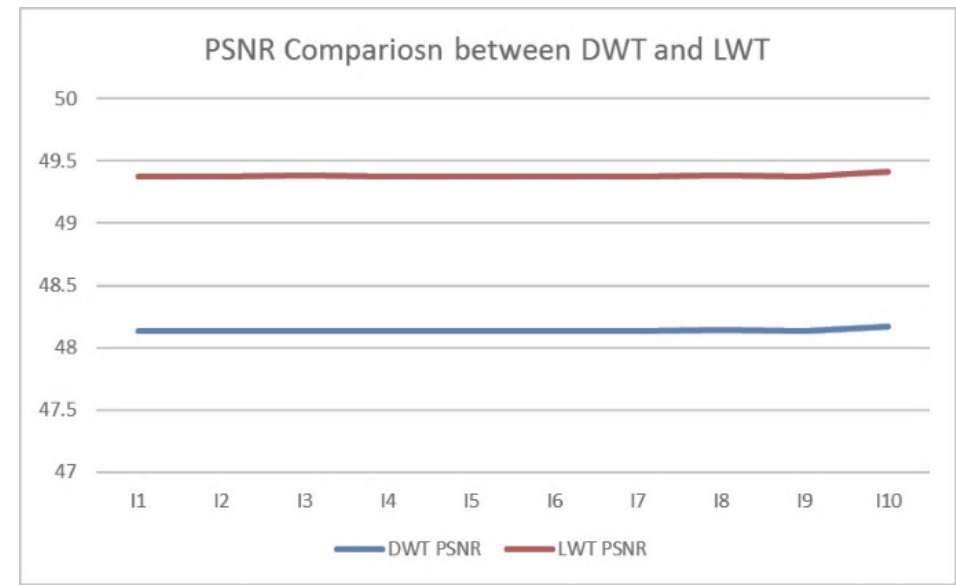

Figure 7. PSNR comparison

\subsection{Robustness evaluation}

The quality of the watermarked images is tested and compared for both transforms. The watermark is recovered from watermarked images after different geometric attacks; JPG compression with 70\% and $50 \%$, salt and pepper with two intensities 0.01 and 0.005 , LPF, sharpening, cropping $25 \%$, and rotation by 0.02 . Two evaluation metrics are used, the normalized correlation coefficient (NCC) and the bit error rate (BER). The NCC is give according to the following equation:

$$
N C C=\frac{\sum_{i}^{m} \sum_{j}^{n}\left[W_{i j} W^{\prime}{ }_{i j}\right]}{\sqrt{\sum_{i}^{m} \sum_{j}^{n}\left(W m_{i j}\right)^{2}} \sqrt{\sum_{i}^{m} \sum_{j}^{n}\left(W m^{\prime}{ }_{i j}\right)^{2}}}
$$

$\mathrm{Wm}, \mathrm{Wm}$ ' are original and recovered watermark, respectively each of size $\mathrm{m} \times \mathrm{n}$. 
BER, on the other hand is given as follows:

$$
B E R=\frac{1}{m \times n} \sum_{i}^{m} \sum_{j}^{n}\left[W_{i j} \otimes W_{i j}^{\prime}\right] \times 100 \%
$$

$\mathrm{Wm}, \mathrm{Wm}$ ' are original and recovered watermark, respectively each of size $\mathrm{m} \times \mathrm{n}$.

Figure 8, summarizes the average of the NCC after all attacks for tested images and the average is taken for the embedding in both transforms. Figure 9 shows the average of BER for the same images, attacks and transforms.

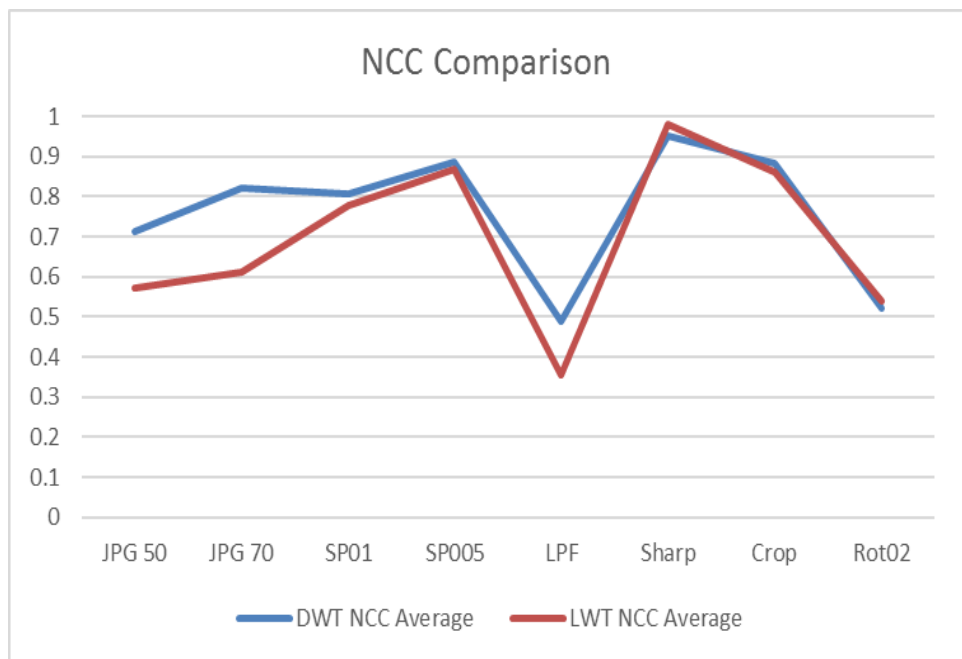

Figure 8. NCC comparison between DWT and LWT

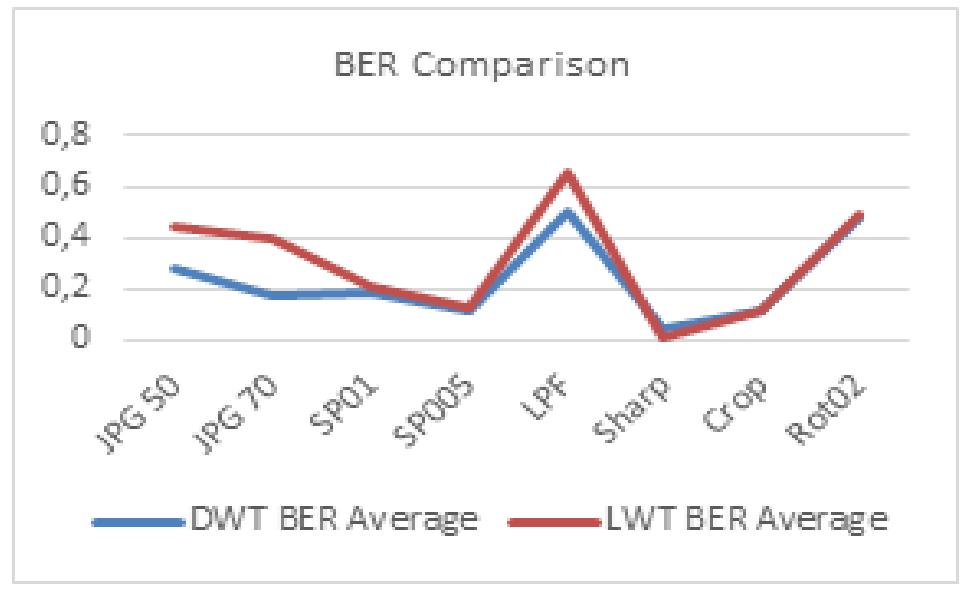

Figure 9. BER comparison between DWT and LWT

Details of the NCC, BER and the extracted watermark for each attack independently for DWT and LWT- based extracted watermarks are shown in Tables 3 and 4 respectively. From the results, DWT-based watermarking produces watermarks with higher NCC and less BER for most attacks, which indicate that DWT is more robust than LWT in this context. In general, the nature of DWT which based on Fourier transform, has more intensive changes on images, so it is irreversible. Accordingly the quality of the watermarked images is less, but the robustness of the watermarked images is higher. 
Table 3. NCC and BER for DWT- based extracted watermark

\begin{tabular}{|c|c|c|c|c|c|c|c|c|c|}
\hline Image & & JPG 50 & JPG 70 & SP01 & SP005 & LPF & Sharp & Crop & Rot02 \\
\hline \multirow{2}{*}{1} & $\mathrm{NCC}$ & 0.7225 & 0.8171 & 0.8016 & 0.8811 & 0.4769 & 0.9479 & 0.8967 & 0.5112 \\
\hline & BER & 0.2764 & 0.1870 & 0.1997 & 0.1233 & 0.5090 & 0.0520 & 0.1169 & 0.4778 \\
\hline \multirow{2}{*}{2} & NCC & 0.7426 & 0.8413 & 0.8107 & 0.8656 & 0.4595 & 0.8656 & 0.8784 & 0.5391 \\
\hline & BER & 0.2673 & 0.1628 & 0.1963 & 0.1392 & 0.5269 & 0.1392 & 0.1289 & 0.4756 \\
\hline \multirow{2}{*}{3} & $\mathrm{NCC}$ & 0.5624 & 0.7426 & 0.7993 & 0.8839 & 0.5002 & 0.9479 & 0.8839 & 0.5162 \\
\hline & BER & 0.4172 & 0.2278 & 0.1980 & 0.1113 & 0.4873 & 0.0530 & 0.1162 & 0.4761 \\
\hline \multirow{2}{*}{4} & $\mathrm{NCC}$ & 0.7083 & 0.8336 & 0.8121 & 0.8935 & 0.4801 & 0.9657 & 0.8816 & 0.5075 \\
\hline & BER & 0.2871 & 0.1648 & 0.1824 & 0.1060 & 0.5051 & 0.0281 & 0.1326 & 0.5024 \\
\hline \multirow{2}{*}{5} & NCC & 0.7261 & 0.8230 & 0.8198 & 0.8916 & 0.4975 & 0.9954 & 0.8793 & 0.4943 \\
\hline & BER & 0.2727 & 0.1697 & 0.1819 & 0.1042 & 0.4929 & 0.0034 & & 0.5049 \\
\hline \multirow{2}{*}{6} & $\mathrm{NCC}$ & 0.7499 & 0.8157 & 0.7897 & 0.8834 & 0.4879 & 0.9 & 0.86 & 0.5496 \\
\hline & BER & 0.2527 & 0.1777 & 0.1953 & 0.1162 & 0.4980 & 0.0283 & 0.1331 & 0.4543 \\
\hline \multirow{2}{*}{7} & $\mathrm{NCC}$ & 0.7289 & 0.8217 & 0.7965 & 0.8907 & 0.5162 & 0.5 & 34 & 0.5158 \\
\hline & BER & 0.2732 & & 0.1985 & & 0.4783 & 0.0139 & 0.1 & 0.4895 \\
\hline \multirow{2}{*}{8} & $\mathrm{NCC}$ & 0.7494 & 0.8482 & 0.8208 & 0.8884 & 0.4920 & 080 & 08 & 0.5556 \\
\hline & BER & 0.2520 & 0.1594 & 0.1912 & 0.1157 & 0.4998 & 0.1118 & 0.1240 & 0.4500 \\
\hline \multirow{2}{*}{9} & $\mathrm{NCC}$ & 0.7302 & 0.8258 & 0.8125 & 0.8930 & 0.4778 & 0.9675 & 0.8903 & 0.5103 \\
\hline & BER & 0.2708 & 0.1670 & 0.1926 & 0.1111 & 0.5046 & 0.0322 & 0.1199 & 0.4941 \\
\hline \multirow{2}{*}{10} & NCC & 0.7270 & 0.8331 & 0.8176 & 0.8999 & 0.4865 & 0.9749 & 0.8884 & 0.5080 \\
\hline & BER & 0.2705 & 0.1655 & 0.1873 & 0.1055 & 0.5020 & 0.0229 & 0.1196 & 0.4929 \\
\hline
\end{tabular}


Table 4. NCC and BER for LWT- based extracted watermark

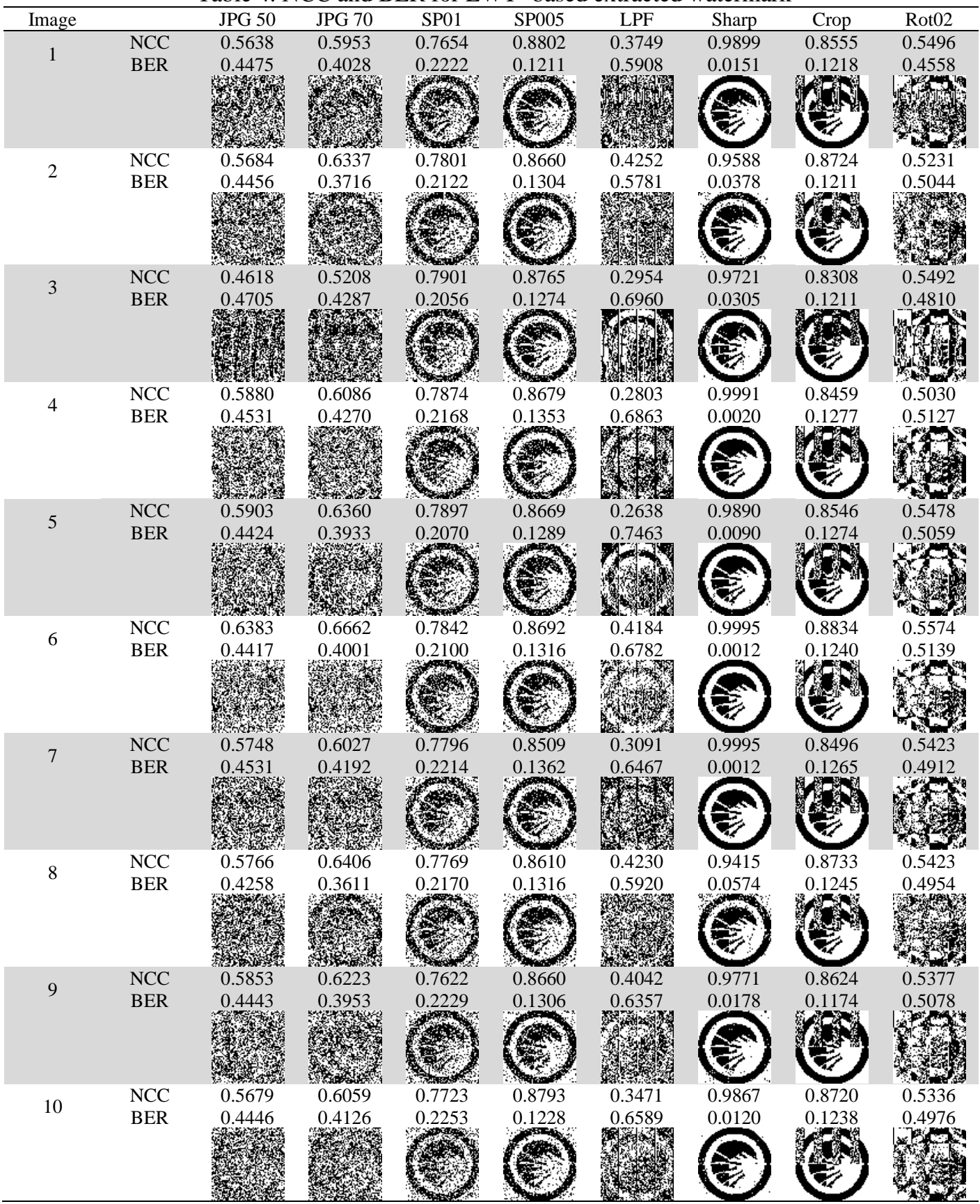

\section{CONCLUSION}

In this paper, a comparison of the performance between the discrete wavelet transform (DWT) and the second generation of wavelet lifting wavelet transform (LWT) in the watermarking process is initiated. The same watermark with the same intensity is embedded with the middle frequency bands of both transforms. The experimental results show that LWT precedes the DWT in terms of objective image quality represented by PSNR. In term of robustness, which is measured by NCC and BER, DWT watermarked images has better robustness than LWT watermarked images after applying different attacks as JPG compression and LPF. The experimental tests produced in this paper can be exploited for creating better image watermarking algorithms using both attempts by considering the advantages of each transformation and combine them. A future work is to create a watermarking algorithm based on both transformations. 


\section{REFERENCES}

[1] Ernawan, F., Liew, S. C., Mustaffa, Z., \& Moorthy, K., "A blind multiple watermarks based on human visual characteristics," International Journal of Electrical and Computer Engineering, vol. 8, no. 4, pp. 2578-1587, 2018.

[2] Meerwald, P., \& Uhl, A., "Survey of wavelet-domain watermarking algorithms," In Security and Watermarking of Multimedia Contents III, International Society for Optics and Photonics, vol. 4314, pp. 505-517, 2001.

[3] Ernawan, F., \& Ariatmanto, D., "Image watermarking based on integer wavelet transform-singular value decomposition with variance pixels," International Journal of Electrical and Computer Engineering, vol. 9, no. 3, pp. 2185, 2019.

[4] Prajwalasimha S N, Chethan Suputhra S, Mohan C S., "Performance analysis of DCT and successive division based digital image watermarking scheme," Indonesian Journal of Electrical Engineering and Computer Science. vol. 15, no. 2, 2019.

[5] Barni, M., Bartolini, F., \& Piva, A., "Improved wavelet-based watermarking through pixel-wise masking," IEEE transactions on image processing, vol. 10, no. 5, pp. 783-791, 2001.

[6] Sweldens, W, "Lifting scheme: a new philosophy in biorthogonal wavelet constructions," In SPIE's 1995 International Symposium on Optical Science, Engineering, and Instrumentation. International Society for Optics and Photonics, pp. 68-79, September, 1995.

[7] Hannoun, K., Hamiche, H., Lahdir, M., Laghrouche, M., \& Kassim, S. "A Novel DWT Domain Watermarking Scheme Based On a Discrete-Time Chaotic System," IFAC-PapersOnLine, vol. 51, no. 33, 50-55, 2018.

[8] Abdulrahman, A. K., \& Ozturk, S. "A novel hybrid DCT and DWT based robust watermarking algorithm for color images." Multimedia Tools and Applications, pp. 1-23, 2019.

[9] Laskar, R. H., Choudhury, M., Chakraborty, K., \& Chakraborty, S, "A joint DWT-DCT based robust digital watermarking algorithm for ownership verification of digital images," In Computer networks and intelligent computing, Springer, Berlin, Heidelberg, pp. 482-491, 2011.

[10] Lu, W., Sun, W., \& Lu, H. "Robust watermarking based on DWT and nonnegative matrix factorization," Computers \& Electrical Engineering, vol. 35, no. 1, pp. 183-188, 2009.

[11] Keshavarzian, R., \& Aghagolzadeh, A. "ROI based robust and secure image watermarking using DWT and Arnold map," AEU-International Journal of Electronics and Communications, vol. 70, no. 3, pp. 278-288, 2016.

[12] Hannoun, K., Hamiche, H., Lahdir, M., Laghrouche, M., \& Kassim, S., "A Novel DWT Domain Watermarking Scheme Based On a Discrete-Time Chaotic System," IFAC-PapersOnLine, vol. 51, no. 33, 50-55, 2018.

[13] Araghi, T. K., \& Manaf, A. A., "An enhanced hybrid image watermarking scheme for security of medical and nonmedical images based on DWT and 2-D SVD," Future Generation Computer Systems, pp. 1223-1246, 2019.

[14] Kabra, R. G., \& Agrawal, S. S., "Robust embedding of image watermark using LWT and SVD," In Communication and Signal Processing (ICCSP), International Conference on IEEE, pp. 1968-1972, April 2016.

[15] Ftima, S. B., Talbi, M., \& Ezzedine, T., "LWT-SVD secure image watermarking technique," In Electronics, Communication and Aerospace Technology (ICECA), 2017 International conference of IEEE. vol. 1, pp. 510-517, April 2017.

[16] Islam, M., Roy, A., \& Laskar, R. H., "SVM-based robust image watermarking technique in LWT domain using different sub-bands," Neural Computing and Applications, pp. 1379-1403, 2018.

[17] Tomar, V., Kumar, A., \& Choudhary, A., "Conception \& implementation of a novel Digital image watermarking algorithm using cascading of DCT and LWT," International Conference on Reliability Optimization and Information Technology (ICROIT), pp. 501-505, 2014.

[18] Preet, C., \& Aggarwal, R. K., "Multiple image watermarking using LWT, DCT and arnold transformation," International Conference on Trends in Electronics and Informatics (ICEI), pp. 158-162, 2017.

[19] Taha, T. B., Ngadiran, R., \& Ehkan, P., "Adaptive Image Watermarking Algorithm Based on an Efficient Perceptual Mapping Model," IEEE Access, 6, 66254-66267, 2018.

[20] Saini, M., "LWT Based Hybrid Digital Watermarking Scheme in YCbCr Colour Space," International Conference on Intelligent Circuits and Systems (ICICS), pp. 206-211, IEEE, April 2018.

[21] Singh, S. P., \& Bhatnagar, G., "A simplified watermarking algorithm based on lifting wavelet transform," Multimedia Tools and Applications, vol. 78, no. 2, pp. 1-22, 2019.

[22] Gholipour, M., "Design and implementation of lifting based integer wavelet transform for image compression applications," In International Conference on Digital Information and Communication Technology and Its Applications. Springer, Berlin, Heidelberg. pp. 161-172, June 2011,

[23] Taha, T. B., Ngadiran, R., Ehkan, P., \& Sultan, M. T., "Image tamper detection and recovery using lifting schemebased fragile watermarking," Journal of theoretical \& applied information technology, vol. 96, no. 8, 2018.

[24] Wang, Z., Bovik, A. C., Sheikh, H. R., \& Simoncelli, E. P., "Image quality assessment: from error visibility to structural similarity," IEEE transactions on image processing, vol. 13, no. 4, pp. 600-612, 2004.

[25] Silpa, K., \& Mastani, S. A., "Comparison of image quality metrics," Int. J. Eng. Res. Technol, vol. 1, no. 4, 2012

[26] Kotevski, Z., \& Mitrevski, P., "Experimental Comparison of PSNR and SSIM Metrics for Video Quality Estimation,” In ICT Innovations, Springer, Berlin, Heidelberg, pp. 357-366, 2009. 